\title{
A standardized boundary element method volume conductor model
}

\author{
Manfred Fuchs ${ }^{a}$,*, Jörn Kastner ${ }^{a}$, Michael Wagner ${ }^{a}$, Susan Hawes ${ }^{b}$, John S. Ebersole ${ }^{b}$ \\ ${ }^{a}$ Neuroscan Laboratories, Lutterothstrasse 28e, D-20255 Hamburg, Germany \\ ${ }^{\mathrm{b}}$ University of Chicago, Chicago, IL 60637, USA
}

Accepted 23 January 2002

\begin{abstract}
Objectives: We used a 3-compartment boundary element method (BEM) model from an averaged magnetic resonance image (MRI) data set (Montreal Neurological Institute) in order to provide simple access to realistically shaped volume conductor models for source reconstruction, as compared to individually derived models. The electrode positions were transformed into the model's coordinate system, and the best fit dipole results were transformed back to the original coordinate system. The localization accuracy of the new approach was tested in a comparison with simulated data and with individual BEM models of epileptic spike data from several patients.

Methods: The standard BEM model consisted of a total of 4770 nodes, which describe the smoothed cortical envelope, the outside of the skull, and the outside of the skin. The electrode positions were transformed to the model coordinate system by using 3-5 fiducials (nasion, left and right preauricular points, vertex, and inion). The transformation consisted of an averaged scaling factor and a rigid transformation (translation and rotation). The potential values at the transformed electrode positions were calculated by linear interpolation from the stored transfer matrix of the outer BEM compartment triangle net. After source reconstruction the best fit dipole results were transformed back into the original coordinate system by applying the inverse of the first transformation matrix.

Results: Test-dipoles at random locations and with random orientations inside of a highly refined reference BEM model were used to simulate noise-free data. Source reconstruction results using a spherical and the standardized BEM volume conductor model were compared to the known dipole positions. Spherical head models resulted in mislocation errors at the base of the brain. The standardized BEM model was applied to averaged and unaveraged epileptic spike data from 7 patients. Source reconstruction results were compared to those achieved by 3 spherical shell models and individual BEM models derived from the individual MRI data sets. Similar errors to that evident with simulations were noted with spherical head models. Standardized and individualized BEM models were comparable.

Conclusions: This new approach to head modeling performed significantly better than a simple spherical shell approximation, especially in basal brain areas, including the temporal lobe. By using a standardized head for the BEM setup, it offered an easier and faster access to realistically shaped volume conductor models as compared to deriving specific models from individual 3-dimensional MRI data. (C) 2002 Elsevier Science Ireland Ltd. All rights reserved.
\end{abstract}

Keywords: Boundary element method; EEG; Triangle refinement; Source reconstruction; Localization accuracy

\section{Introduction}

The boundary element method (BEM) improves the localization accuracy of bioelectric source reconstruction results, as compared to simple spherical shell models, by approximating the volume conductor properties of realistically shaped compartments of isotropic and homogeneous conductivities. The anisotropy and fine structure of the real tissue surrounding the electric sources to be reconstructed are purposefully neglected. These factors can in principle be treated by finite element methods (FEM), but they suffer from large computational effort and in vivo conductivity and anisotropy para-

\footnotetext{
* Corresponding author. Tel.: +49-40-4018-9944; fax: +49-40-40189949.

E-mail address: mfuchs@neuroscan.com (M. Fuchs).
}

meters that are mostly unknown. The BEM is a compromise between over-simplified, spherically symmetric models, that reflect only mean conductivities but not the shape of the compartments, and overly complex models for which detailed real tissue data are not available (Geddes and Baker, 1963; Law, 1993; van Burik and Peters, 2000).

Many authors have already discussed and improved the BEM (Geselowitz, 1967; Hämäläinen and Sarvas, 1989; Meijs et al., 1989; Oostendorp and van Oosterom, 1989; Cuffin, 1990; Fletcher et al., 1995; Yvert et al., 1995; Ferguson and Stroink, 1997; Fuchs et al., 1998, 2001; Musha and Okamoto, 1999; Frijns et al., 2000). The BEM requires a description of the compartment surfaces by closed triangle meshes with a limited number of nodes. It is limited by the computational power and the memory requirement for storing the huge BEM system matrix. The matrix size is propor- 
tional to the square of the total number of nodes, and the computational effort to decompose the BEM matrix is proportional to the third power of the number of nodes, whereas the accuracy of the BEM is roughly proportional to the number of nodes representing the realistic model. The computation time needed for a forward calculation of the electric potential distribution at the given electrode positions is also proportional to the number of nodes and to the number of electrodes.

In this investigation, we developed a standardized BEM model (sBEM) from averaged magnetic resonance imaging (MRI) data (Montreal Neurological Institute), having computed and stored the transfer matrix for all nodes of the outermost (skin) compartment. The measured electrode positions were transformed and scaled to the sBEM model coordinate system, which is aligned by the PreAuricular points and the Nasion (PAN system). The electric potential values at the transformed electrode positions were calculated by linear interpolation from the nodes of the sBEM model skin compartment. Finally the source reconstruction results were transformed back to the original electrode coordinate system by applying the inverse transformations.

By doing so we eliminated the need to segment an individual subject's anatomical data into the 3 main BEM model compartments, which requires sophisticated algorithms or manual interaction. If overlay of the source reconstruction results with the individual anatomy is not required, the subject's anatomical image data are not at all needed. Furthermore the time consuming BEM matrix setup and decomposition steps can be omitted. Thus an easier and much faster access to realistically shaped volume conductor models can be achieved.

\section{Methods}

\subsection{The boundary element method}

The boundary element method (BEM) allows to calculate the electric potential $V$ of a current source in an inhomogeneous conductor by solving the following integral equation, if the conducting object is divided by closed surfaces $S_{i}$ $\left(i=1, \ldots, n_{\mathrm{s}}\right)$ into $n_{s}$ compartments, each having a different enclosed isotropic conductivity $\sigma_{j}^{\text {in }}$. The electric potential at position $\underline{r} \in S_{k}$ is then given by (Geselowitz, 1967; Sarvas, 1987):

$\bar{\sigma}_{k} V(\underline{r})=\sigma_{0} V_{0}(\underline{r})+\frac{1}{4 \pi} \sum_{i=1}^{n_{s}} \Delta \sigma_{i} \oiint_{S_{i}} V\left(\underline{r}^{\prime}\right) \underline{n}\left(\underline{r}^{\prime}\right) \cdot \frac{\underline{r}^{\prime}-\underline{r}}{\left|\underline{r}^{\prime}-\underline{r}\right|^{3}} \mathrm{~d} S_{i}$

with $V_{0}$ representing the potential of the source in an unlimited homogeneous medium with conductivity $\sigma_{0}$, the mean conductivity $\bar{\sigma}_{k}=\left(\sigma_{k}^{\text {in }}+\sigma_{k}^{\text {out }}\right) / 2$, and the conductivity differences $\Delta \sigma_{i}=\sigma_{i}^{\text {in }}-\sigma_{i}^{\text {out }}$. To calculate the electric fields it is necessary to approximate numerically the two integrals over the closed surfaces $S_{i}$ of the conductor boundaries consisting of differential surface elements $\left(\mathrm{d} S_{i}^{\prime}\right)$ and with surface normal orientations $\underline{n}$ at positions $\underline{r}^{\prime}$. The surfaces are described by a large number of small triangles and the integrals are replaced by summations over these triangle areas. Different assumptions about the variation of the potential over the triangle area can be applied (van Oosterom and Strackee, 1983; de Munck, 1992; Ferguson et al., 1994; Schlitt et al., 1995; Fuchs et al., 1998): averaged, regionally constant, linear, and quadratic dependencies. The potential values or the coefficients of the basis functions used to approximate the potentials on the surface elements form a vector of unknowns, which can be solved through the following matrix formulation:

$\underline{\underline{\sigma}} \underline{V}=\sigma_{0} \underline{V}_{0}+\underline{B} \underline{V} \Rightarrow \underline{V}=(\underline{\underline{\bar{\sigma}}}-\underline{\underline{B}})^{-1} \sigma_{0} \underline{V}_{0}$

If one explicitly solves Eq. (2) just for the fixed number of measurement positions, a transfer matrix $\underline{\underline{T}}$ is obtained, that relates the sensor signals to the homogeneous potentials (e.g. Fletcher et al., 1995). The potential vector $\underline{V}$, that contains the field distribution at all skin nodes, generated by a (dipolar) source inside the innermost compartment (brain) can thus be easily computed by a simple matrixvector multiplication:

$\underline{V}=\underline{T} \underline{V}_{0} \quad$ with $\quad \underline{\underline{T}}=(\underline{\underline{\bar{\sigma}}}-\underline{\underline{B}})^{-1} \sigma_{0}$

The column vector $\underline{V}_{0}$ contains the electric potential values $V_{0 i}$ of all skin-nodes $i$ at position $\underline{r}_{i}$ for the source in an infinite homogeneous conductor with conductivity $\sigma_{0}$ (dipole at position $\underline{r}_{j}$, current $j_{-}$:

$V_{0 i}=\frac{1}{4 \pi \sigma_{0}} j \frac{\underline{r}_{i}-\underline{r}_{j}}{\left|\underline{r}_{i}-\underline{r}_{j}\right|^{3}}$

\subsection{A standardized boundary element method model}

A standardized boundary element method (sBEM) volume conductor model was derived from an averaged T1-weighted magnetic resonance image (MRI) dataset, which is available from the Montreal Neurological Institute (MNI, www.mni.mcgill.ca). This 3-dimensional (3D) dataset contains 91 axial slices with $91 \times 109$ pixels and a voxelsize of $2 \times 2 \times 2 \mathrm{~mm}^{3}$. The 3 compartments of the sBEMmodel were segmented from the 3D-dataset by a gray level threshold controlled 3D region growing algorithm described earlier (Wagner et al., 1995, 1997; Fuchs et al., 1998). The results of the segmentation processes are displayed in Fig. 1. The skin, skull, and brain compartments are triangulated using mean triangle side lengths (node distances) of 8.3, 6.1 , and $4.4 \mathrm{~mm}$ resulting in 2062, 2774, and 4692 triangles (1033, 1389, and 2348 nodes or 9528 triangles and 4770 nodes overall). The tessellations of the 3 shells are depicted in Fig. 2.

The triangles that represent the compartment boundaries were globally and virtually refined (no real nodes were added, which would increase the computational effort) and 


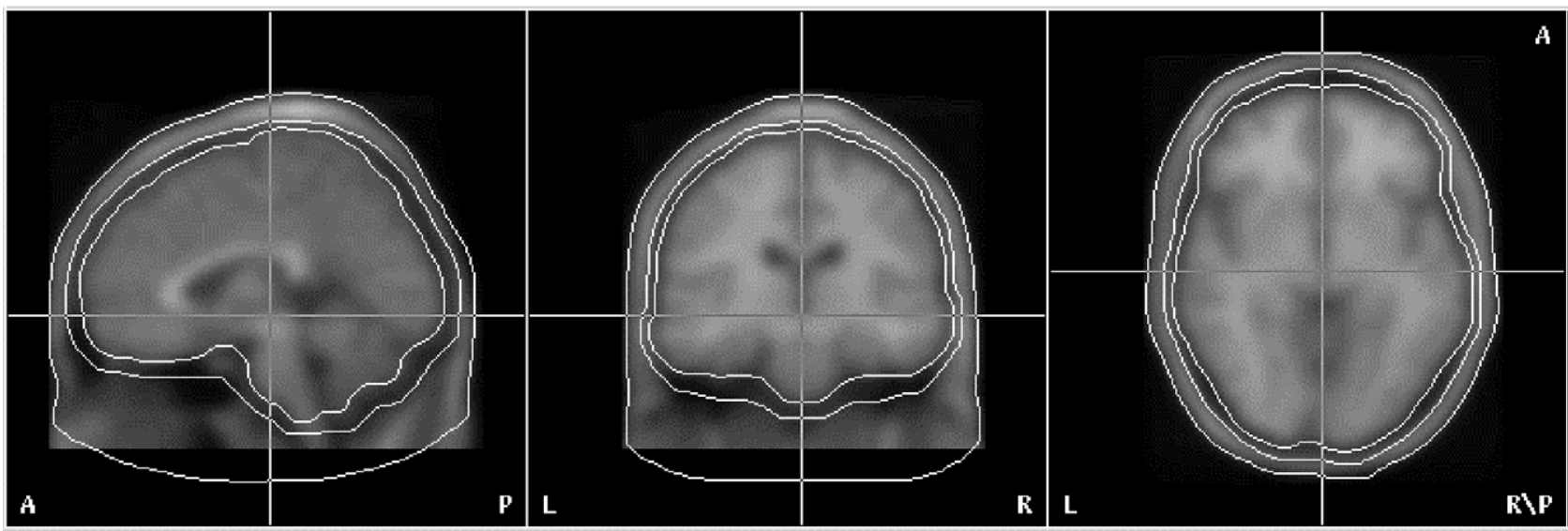

Fig. 1. Orthogonal slices through the averaged 3D MRI dataset (Montreal Neurological Institute) with the 3 segmented sBEM compartments. The skin compartment is manually smoothed and extended at the bottom.

a regionally constant potential approximation over the subtriangles was chosen. This approach results in better accuracy than the standard linear potential approximation (de Munck, 1992) with comparable computational effort (Fuchs et al., 1998, 2001). The size of the BEM matrix stays the same (total number of nodes is unchanged), only during set up of the system matrix the solid angle computation is slightly more demanding. In order to further improve the model accuracy, the isolated problem approach (IPA) (Hämäläinen and Sarvas, 1989) was used.

From the decomposed BEM matrix $\underline{B}$ the transfer matrix $\underline{T}$ for all 1033 nodes of the outermost compartment (skin) is $\overline{\bar{c}}$ omputed and stored (Eq. (3)).

All these initial computations were performed using the Curry V4.5 software package (Neuroscan).

\subsection{Coordinate system matching}

In order to match the coordinate system of the electrodes

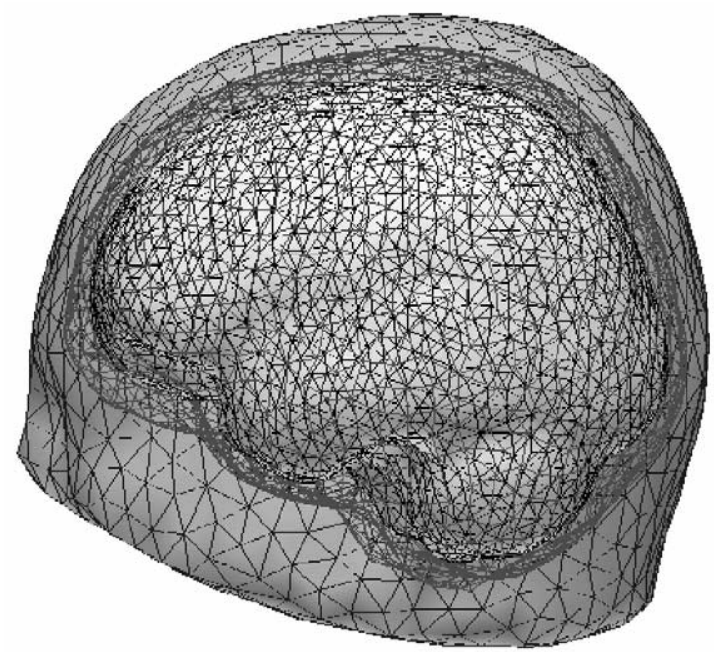

and landmarks with the coordinate system of the sBEM model, both systems were transformed into PAN systems (PreAuricular points and Nasion). In the PAN system the $x$ axis points from the left to the right preauricular point, and the coordinate system origin is in the middle between both preauricular points. The $y$-axis points towards the nasion (posterior - anterior direction) and lies in the plane of all 3 points. Thus, with asymmetric head shapes the nasion's $x$ coordinate is not always at $x=0$. Finally, the $z$-axis points from the bottom to the top towards the vertex at right angles between both $x$ - and $y$-axes forming a right handed basis. Details of the coordinate system matching algorithm are described in Appendix A.

In order to improve the match between the sBEM model dimensions and the electrodes a global scaling factor was applied to the electrode positions. It was calculated from the average of the ratios of the nasion-origin distances and the left-right preauricular point distances. The inverse of this factor has to be applied to the reconstruction results before

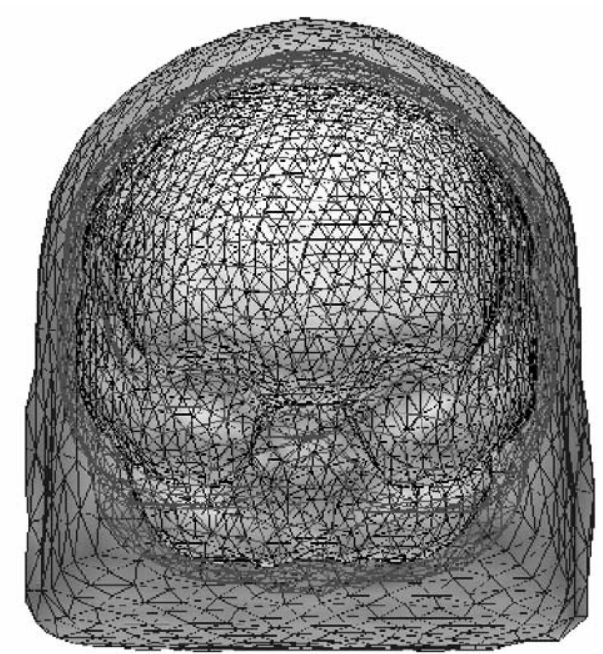

Fig. 2. Standard BEM-model derived from averaged MRI data (Fig. 1). Left and frontal views, 9528 triangles/4770 nodes overall; brain, 2348; skull, 1389; skin, 1033; mean triangle edge sizes (node distances): 4.4, 6.1, $8.3 \mathrm{~mm}$. 
the transformation to the original coordinate system can be performed.

\subsection{Linear interpolation of the electrode potentials}

The transfer matrix Tof the sBEM model (Eq. (3)) was used to calculate the electric potential values of a dipolar source inside the innermost (brain) BEM model compartment at all nodes of the outermost (skin) BEM model compartment. In order to compute the potential values at the measured and transformed electrode positions their locations are projected to the nearest triangle of the skin mesh and a linear interpolation between the 3 potential values of the corresponding triangle nodes is performed (Fig. 3). Details of the linear potential interpolation algorithm are described in Appendix B.

All sBEM model computations are carried out using the Source V2.0 software module (Neuroscan).

\subsection{Simulations}

In order to assess the improvements in source localization accuracy of the sBEM approach as compared to a spherical shell model, simulations using a highly refined BEM model derived from an unaveraged 3D-MR dataset were performed. This reference BEM model consists of 5275 nodes in total (brain/skull/skin compartment: 2720/1350/ 1205; mean node distances: 4.0/6.0/7.4 mm; conductivities: 0.33/0.0042/0.33 S/m). Seventy-one electrodes at extended 10-20 system positions were used to compute the electric field distributions of 4156 randomly oriented test dipoles placed at randomly chosen locations inside the brain compartment.

In order to see the systematic effects of different volume conductor models on dipole localization without random localization errors produced by measurement noise, dipoles were fitted using the noise-free simulated data of the refer-

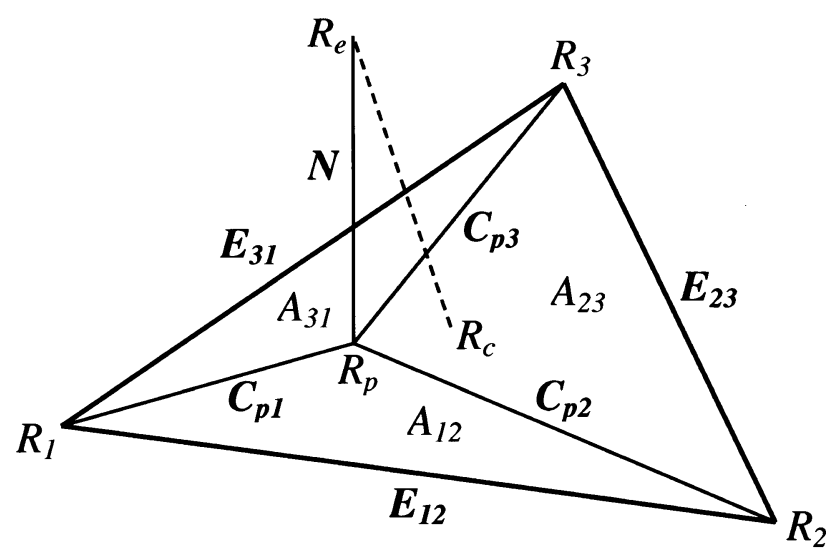

Fig. 3. Linear interpolation of the electric potential at the projected electrode position $R_{\mathrm{p}}$. The electrode is projected to the closest triangle, the weights for the potentials at the triangle nodes are calculated from the ratios of the sub-triangle areas $A_{i j}$ to the total triangle area. ence model. First, a spherical model was set up by fitting a sphere to the electrode positions. This sphere represented the skin compartment, whereas the spherical shells representing the skull and brain compartments were derived by applying relative radii of 93 and $85 \%$. The nonlinear dipole fit procedure (simplex algorithm, Nelder and Mead, 1965) started at the known test dipole positions. Finally the localization error $E$ was determined for each dipole (index $j=1 \ldots N$ ) from the 3 -dimensional distance of the known test-position $P_{j}$ and the fitted dipole position $R_{j}$ and the mean error $M$ is calculated:

$$
\begin{aligned}
& E_{j}=\sqrt{\sum_{i=x, y, z}\left(R_{j i}-P_{j i}\right)^{2}} \\
& M=\frac{1}{N} \sum_{j=1 . . N} E_{j}
\end{aligned}
$$

The same evaluations were then performed using the sBEM model (4770 nodes overall) with the electrodes transformed and matched to the sBEM coordinate system as described above.

The effect of measurement noise to the localization accuracy and stability especially in areas close to the compartment boundaries (Fuchs et al., 2001) is beyond the scope of this work and thus not studied here in more detail.

\subsection{Epileptic spike data}

For a more realistic comparison of individual BEM, standardized BEM, and spherical volume conductor models, 11 averaged epileptic spikes from 7 patients and 12 unaveraged spikes from one such patient were evaluated. Spike potentials that were used for averages were acquired during standard long-term EEG monitoring using 26-32 scalp electrodes (International 10-20 plus bilateral supplementary subtemporal placements (F9, T9, M1, F10, T10, M2) plus selected 10-10 intermediary positions). Averages in a given patient were derived from 3 to 8 individual spikes with the best signal to noise ratio that had the same voltage topography over the time course of the spike. Unaveraged spikes were obtained from one patient during a standard EEG with 21 electrodes.

Two typical spike complexes are shown in Fig. 4 as time courses and as two-dimensional voltage maps. Individual, realistically shaped BEM compartments (Fig. 5) were constructed from T1-weighted MR data of the patients by an automated procedure (Wagner et al., 1995, 1997; Fuchs et al., 1998). The main parameters of all measurements and the individual BEM models used can be found in Table 1.

In order to make comparisons, source reconstructions using standard BEM and spherical shell (outer radius fitted to the electrodes) models were performed, and the 3D localization differences between the source models were evaluated. In order to determine mean localization differences $(E)$, single moving dipoles were fitted at all spike latencies 

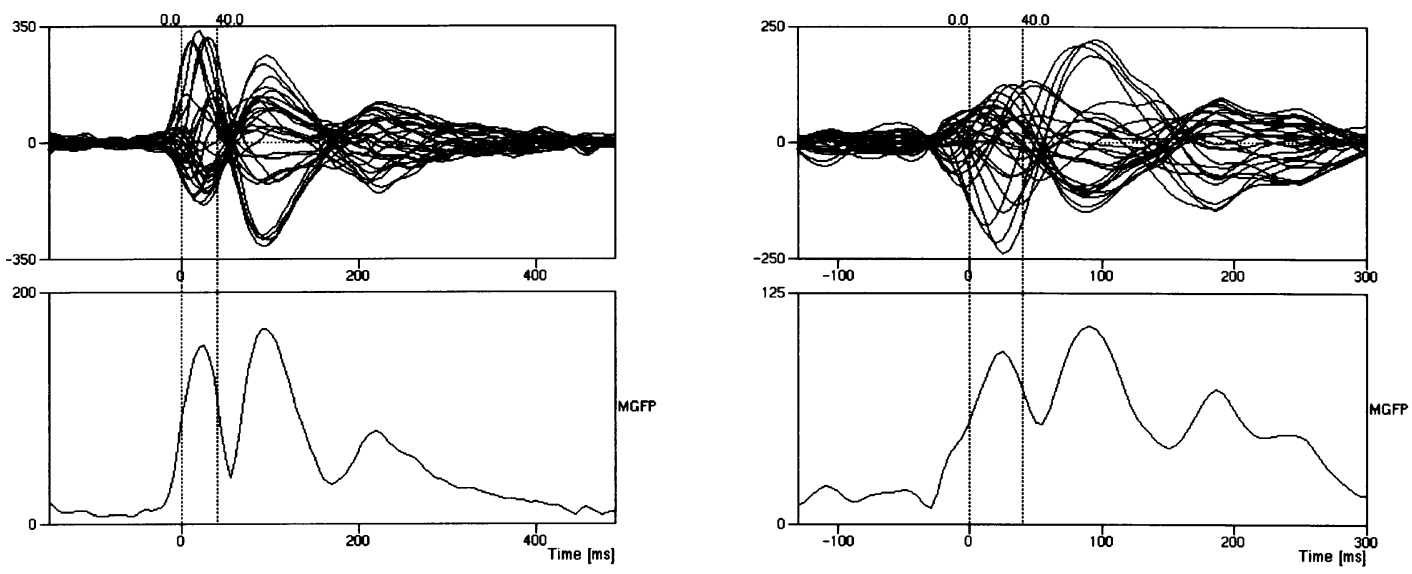

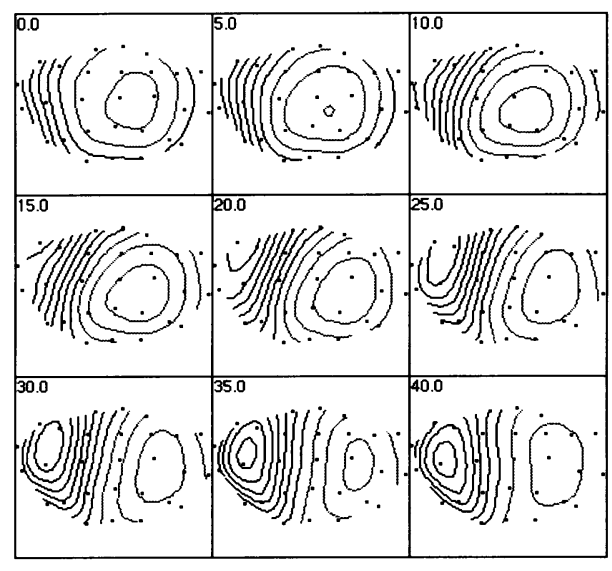

patient 1

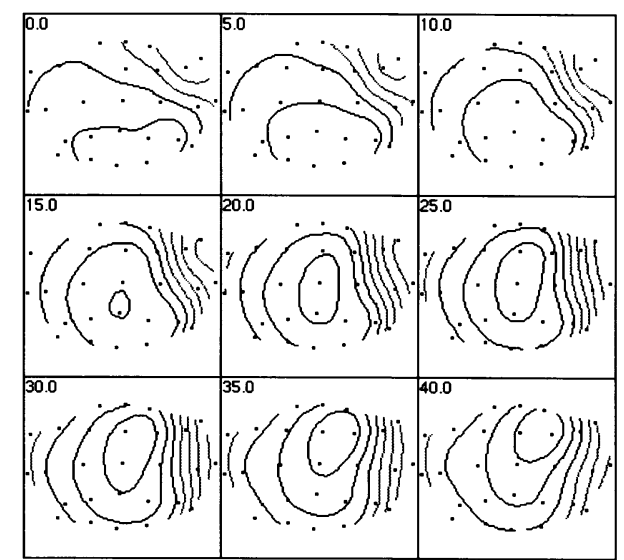

patient 5

Fig. 4. Typical examples of epileptic spike data (spike / wave complexes of patient 1 and 5, latency ranges for source reconstruction are indicated by vertical cursor lines). EEG traces are shown as butterfly plots together with mean global field power (MGFP) and equipotential mappings in top projections.

$\left(t=t_{1}-t_{\mathrm{s}}\right)$ and the 3D distances $\left(E_{t}\right.$, compare Eq. (5)) using different volume conductor models were calculated. These distances $E_{t}$ were weighted by the inverse of the fit quality $F_{t}$ (deviation between measured $M_{k}$ and calculated $C_{k}$ potential values $(k=1-m$ electrodes $)$ ) to emphasize good and to suppress bad matching dipole fits and then averaged. Typically dipole fits are 'good' around the peak latencies of the spike, which exhibit good signal to noise ratios (SNRs).

$E=\sum_{t=t_{1}}^{t_{\mathrm{s}}} \frac{E_{t}}{F_{t}} / \sum_{t=t_{1}}^{t_{\mathrm{s}}} \frac{1}{F_{t}}$ with $F_{t}=\sqrt{\sum_{k=1}^{m}\left(M_{k}-C_{k}\right)^{2}}$

Table 1

Main parameters of the epileptic spike data and the individual BEM models of 7 patients

\begin{tabular}{|c|c|c|c|c|c|c|c|}
\hline Patient & No. of electrodes & No. of spikes & Maximum SNR & No. of nodes & Skin & Skull & Brain \\
\hline $1 \mathrm{a}$ & 28 & 1 & 18 & 3632 & 1281 & 1006 & 1345 \\
\hline $1 b$ & 21 & 12 & 13 & 3632 & 1281 & 1006 & 1345 \\
\hline 2 & 26 & 2 & 16 & 3833 & 1273 & 1099 & 1461 \\
\hline $3 a$ & 32 & 1 & 14 & 4397 & 1274 & 1325 & 1798 \\
\hline $3 b$ & 27 & 1 & 19 & 4448 & 1266 & 1354 & 1828 \\
\hline 4 & 27 & 1 & 12 & 4297 & 1429 & 1199 & 1669 \\
\hline 5 & 27 & 1 & 10 & 4172 & 1226 & 1236 & 1710 \\
\hline 6 & 32 & 1 & 6 & 3563 & 1079 & 1081 & 1403 \\
\hline $7 \mathrm{a}$ & 27 & 1 & 8 & 3824 & 1196 & 1120 & 1508 \\
\hline $7 b$ & 30 & 2 & 8 & 3824 & 1196 & 1120 & 1508 \\
\hline
\end{tabular}



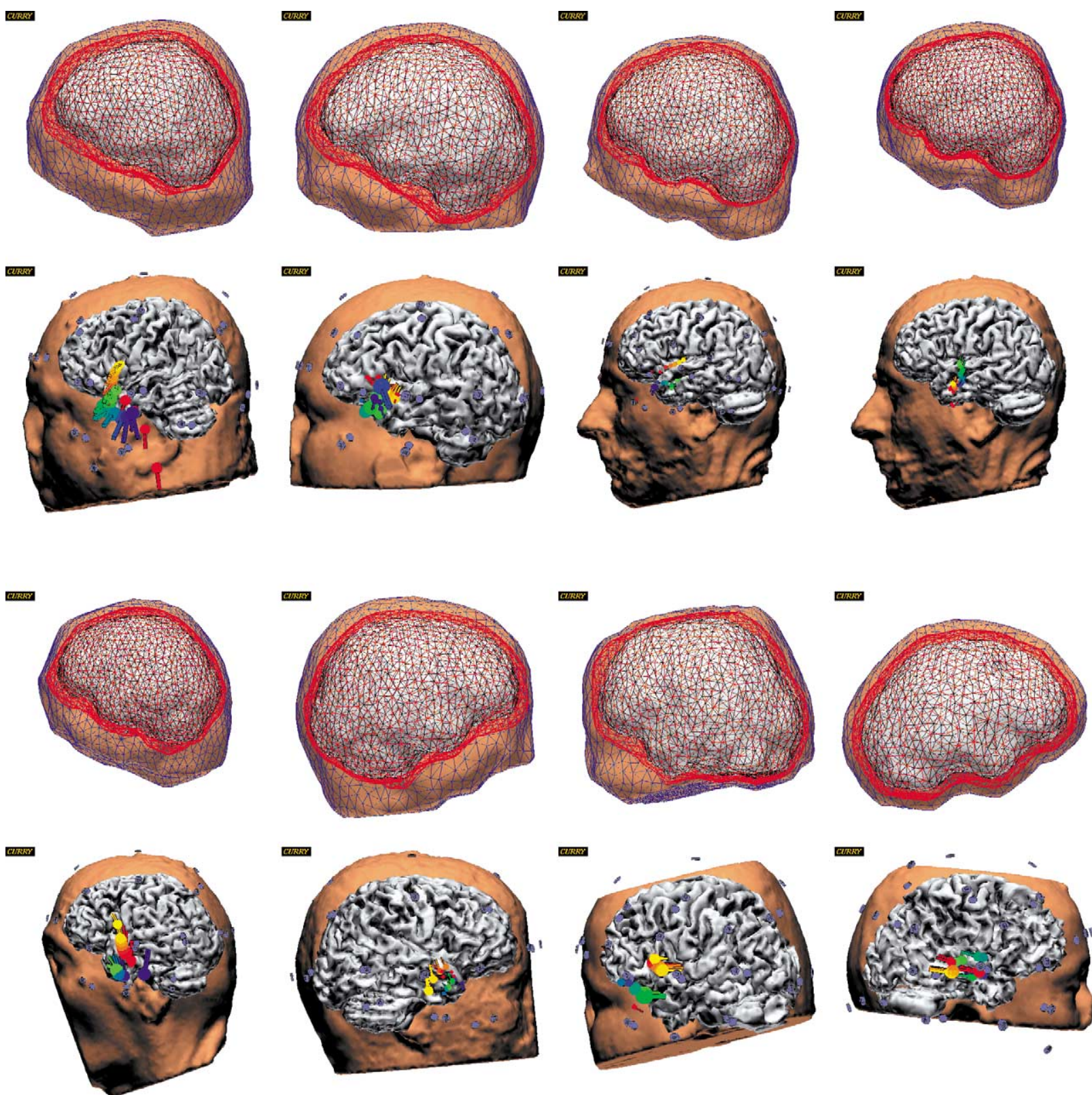

Fig. 5. Eight individual BEM models of 7 patients (compare Table 1) shown together with segmented skin and brain surfaces, electrodes, and dipole reconstruction results as colored symbols (yellow/red, spherical shells; blue/green, BEM volume conductor model). Patients 6 and 7 have incomplete MR data sets resulting in unreliable BEM models (lower right).

\section{Results}

\subsection{Simulations}

The spatial distributions of the localization errors for both sBEM and spherical volume conductor models using simulated data are displayed in Fig. 6. The localization errors are represented by the radii of circles centered at the true positions of the test-dipoles with the reference BEM model. For better visibility the radii are downscaled by a factor of 5 to $20 \%$ of their real size. The mean location errors averaged over all 4156 randomly distributed test-dipole positions are $6.9 \mathrm{~mm}$ for the sBEM model and $10.8 \mathrm{~mm}$ for the 3 spherical shells model. More important than the improvement in the absolute localization accuracy is the more homogeneous spatial distribution of the localization errors with the sBEM model. The spherical model performs best in the more spherical upper parts of the brain, but fails in the lower frontal, central, and temporal lobe areas, which cannot be well represented by the spherical shells. These findings confirm the earlier studies that showed the same behavior (Fuchs et al., 1998, 2001). For the sBEM model no 
systematic trend in the spatial distribution of the localization errors was be found.

\subsection{Epileptic spike data}

The averaged single dipole localization differences between the 3 different volume conductor approximations are depicted in Fig. 7 for of all 23 epileptic spikes that were evaluated. The upper graph displays the spherical volume conductor result versus the individual BEM model results as reference. The localization differences are shown in the PAN coordinate system and are sorted with ascending zdifferences, since this (vertical) direction exhibits the largest deviations that are always positive. In the horizontal directions the differences are less systematically distributed and scatter around the zero line. For the two patients (P6 and P7) with incomplete MR data sets (Fig. 5) the reference BEM models are not reliable, so these results are separated from the others by the vertical line in the upper row of Fig. 7. These data are also excluded from the mean localization difference calculation below.

In the middle row of Fig. 7 the localization differences between spherical and sBEM volume conductor models are displayed. The averaged source reconstruction results are again sorted with ascending $z$-differences, since this direction dominates the localization differences similar to the
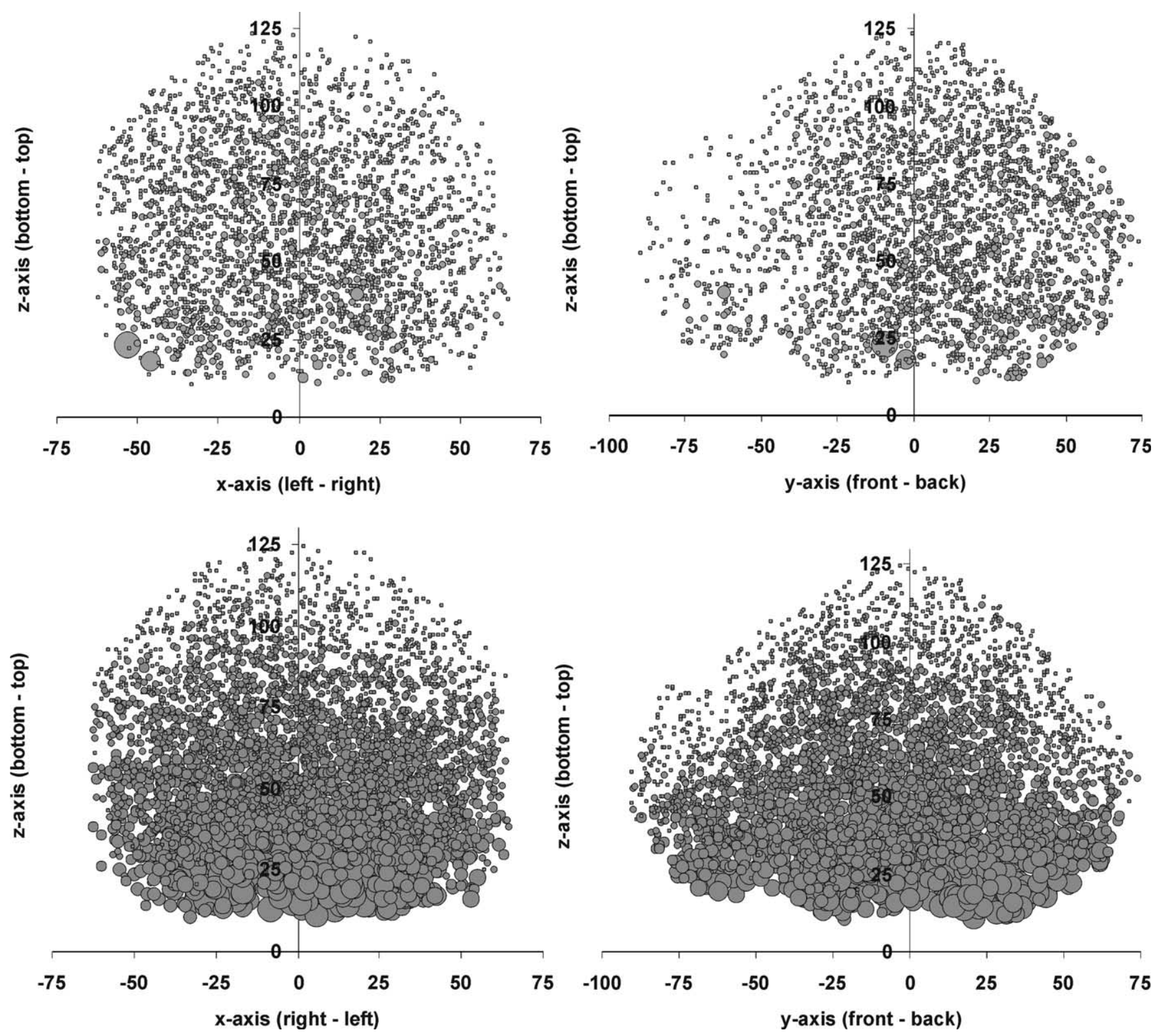

Fig. 6. Dipole localization results using the scaled standard BEM model (upper row) and a spherical shell model (lower row) for simulated data at 4156 randomly chosen positions and orientations inside the brain compartment of a 5275-node reference BEM model. Seventy-one electrodes; average localization errors: standard BEM: $6.9 \mathrm{~mm}$; spherical shells: $10.8 \mathrm{~mm}$. Right column: frontal view. Left column: left side view. Circle radii represent localization errors downscaled to $20 \%$ of their real size for better visibility. 
first case. In this case, all patient data could be evaluated because no individual BEM models were used.

Finally, in the lower row of Fig. 7, the sBEM results are compared to the individual BEM results. No systematic trend in the localization differences can be seen, and accordingly the results are sorted by patient/spike number. The averaged localization differences for spike sources from patients with complete MR data sets and full BEM models are shown in Table 2.

Fig. 8 depicts, in an individual patient example, the significant differences in source localization that occurs when using spherical versus BEM head models and conversely the similarity in source localization between individual and standardized BEM models. Sequential, single 'moving' dipole solutions over the time course of a spike are overlaid onto standardized and individual brain anatomy. Dipoles calculated with spherical head models traverse a large vertical distance and often erroneously reside outside of the temporal lobe, unlike those calculated with either BEM model.
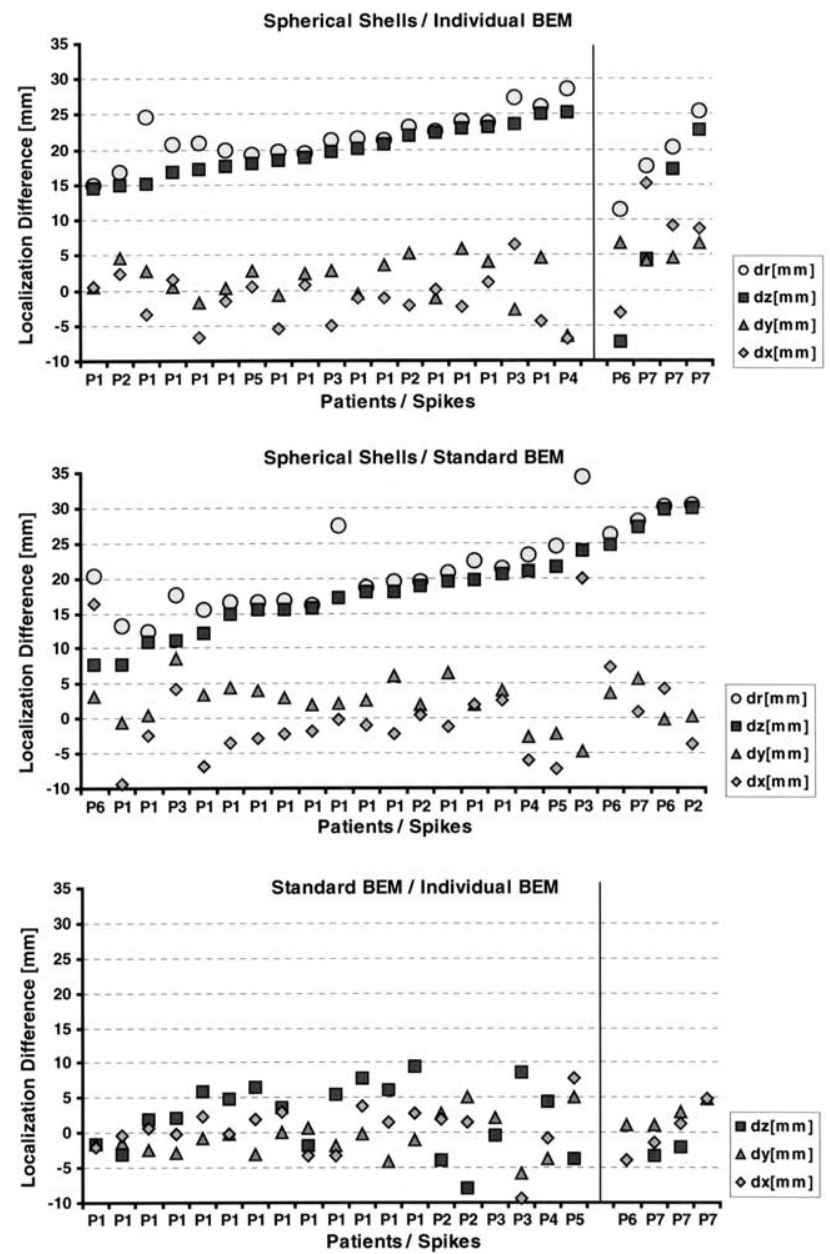

Fig. 7. Averaged dipole localization results of epileptic spike data using individual and scaled standard BEM models are compared to spherical shell models (fitted to the electrodes) and against each other.
Table 2

Mean localization differences of spherical and standard BEM models against individual BEM models averaged over all epileptic spike source reconstruction results

\begin{tabular}{lclcc}
\hline Model comparison & $\mathrm{d} x(\mathrm{~mm})$ & $\mathrm{d} y(\mathrm{~mm})$ & $\mathrm{d} z(\mathrm{~mm})$ & $\mathrm{d} r(\mathrm{~mm})$ \\
\hline Sphere/BEM & -1.4 & 1.4 & 19.9 & 21.9 \\
Sphere/sBEM & 0.3 & 2.3 & 18.4 & 21.4 \\
sBEM/BEM & -0.3 & 0.7 & 2.4 & 8.8 \\
\hline
\end{tabular}

\subsection{Computational performance}

Computational performance was determined with a state-of-the-art PC (AMD Athlon $1000 \mathrm{MHz} / 256 \mathrm{Mb}$ RAM). Using optimized assembler algorithms, the computation of the homogeneous potential $V_{0}$ (Eq. (4)) at all 4770 node positions of the sBEM model took $0.17 \mathrm{~ms}$, and the matrix vector multiplication (Eq. (B10)) required $0.041 \mathrm{~ms}$ per electrode. Thus a forward calculation at 28 electrode positions takes about $1.4 \mathrm{~ms}$. A single dipole fit needs typically 40 iterations of the simplex algorithm (Nelder and Mead, 1965) and including the linear algebra takes about $210 \mathrm{~ms}$, as compared to about $3.4 \mathrm{~ms}$ using an analytically solvable 3 spherical shells model. Accordingly, source reconstructions using the sBEM volume conductor model with about 5000 nodes were about a factor of 62 slower than those using the simplified spherical shells approximation. However, the 5 single dipole fits per second achievable with a modern $\mathrm{PC}$ is adequate in most cases for using sBEM models routinely.

\section{Discussion}

A new realistically shaped volume conductor approximation for EEG source reconstruction is presented. The 3 compartments of the standardized BEM model are segmented from an averaged MR dataset (Figs. 1 and 2). The transfer matrix for all nodes of the skin compartment is stored. The measured electrodes positions are transformed to the sBEM coordinate system by PAN landmarks and an overall scaling factor. The transformed electrode positions are projected to the closest triangles of the outer compartment tessellation and a linear interpolation of the electric potential values at the triangle's nodes is performed (Fig. 3).

The new approach is tested with simulated data from a highly refined reference BEM model with over 4000 randomly distributed and oriented test-dipoles. The localization accuracy is significantly better as compared to the over-simplifying spherical shell approximation, especially in the basal and temporal lobe areas (Fig. 6). In order to test the sBEM model with real measured data, 23 epileptic spikes of 7 patients are evaluated with 3 different volume conductor models and single moving dipole sources. Averaged and weighted dipole locations are compared for individual BEM models, sBEM models, and spherical shell models (Fig. 7 and Table 2). 

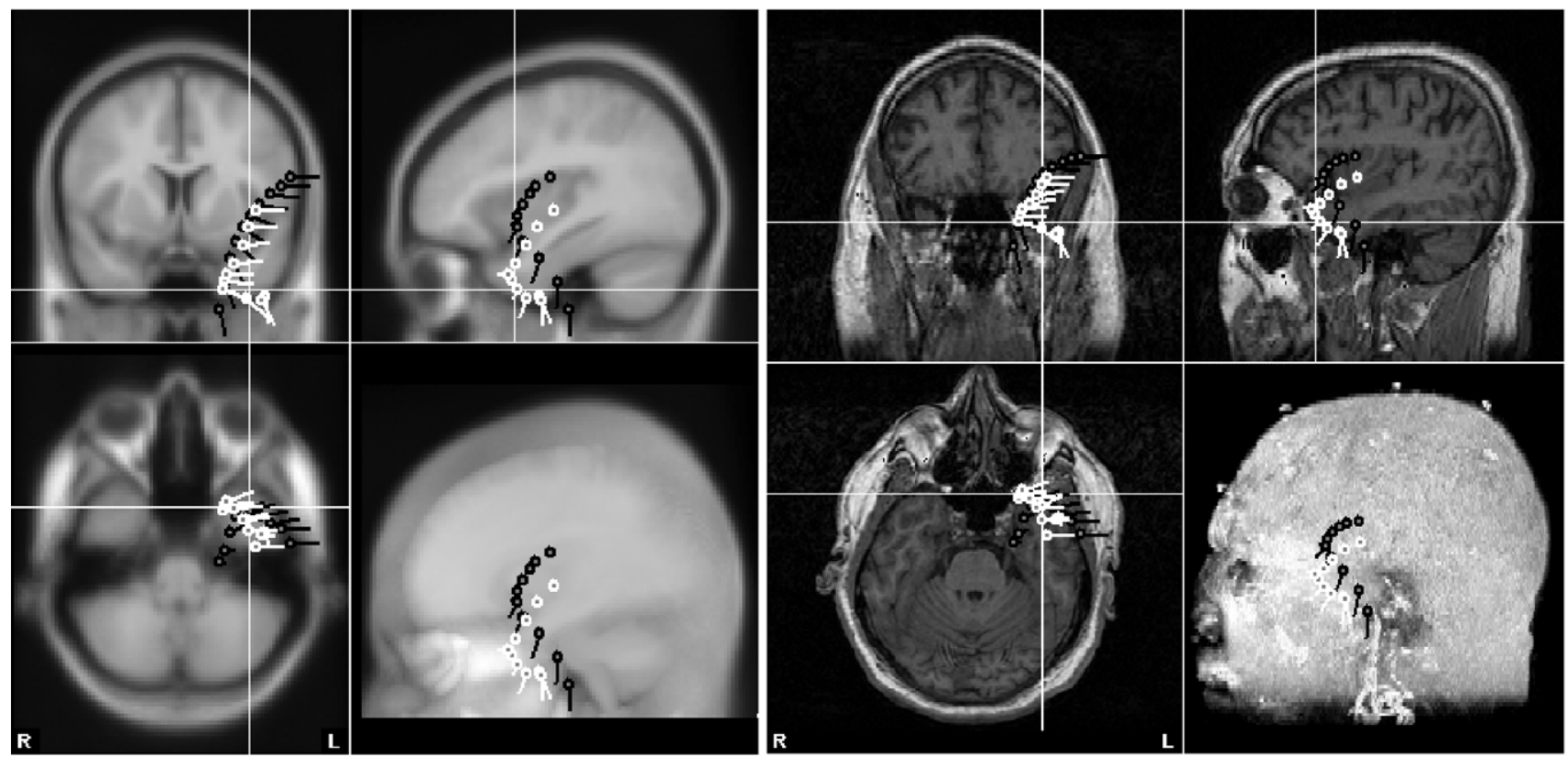

Fig. 8. Single dipole reconstruction results of an epileptic spike (originating from the left temporal lobe) overlaid to the averaged (left) and the individual (right) MR dataset of the patient. Orthogonal slices through the T1-weighted MR data at the cursor positions (white lines) are shown together with maximum intensity projections (MIPs, lower right corners). Single moving dipole results using the spherical volume conductor approximation are shown in black, sBEM model volume conductor results are shown in white. The results for the individual BEM model are not shown, since they differ only slightly from the sBEM model solutions in this case.

The usefulness of advanced EEG analysis in the evaluation of partial epilepsy has been substantiated by numerous recent publications. Dipole source modeling, in particular, has been shown to enhance non-invasive localization of epileptic foci. Several previous studies using simulations, artificial dipoles, or real epileptic spikes have shown that the accuracy of source modeling can be improved by the incorporation of 3D head and brain anatomy for both head models and display of results. Realistic head models of individual patients have seldom been used clinically mainly for practical reasons. In the clinical setting it is not always possible or easy to obtain high quality, thin slice volumetric MRIs on each patient, to devote the time necessary for segmenting brain, skull and scalp surfaces from 3D reconstructions and for performing lengthy calculations required by such models. Until recently this massive computational effort has been a major limiting factor.

In this investigation, we have demonstrated that a practical compromise exists that can provide dramatic improvement in source modeling accuracy without significant increase in effort, namely replacing the standard spherical head model with a standardized realistic head model. By appropriately warping the electrode array of an individual patient to the standardized model, the same realistic head model can be used for any patient with great gain in computational speed because the time-consuming calculations the BEM matrix have already been performed. At the speed of modern PCs the additional computation effort needed to use this standardized model is very reasonable. This is particularly true when considering the improved accuracy afforded by such a standardized model and the amount of effort required to setup an individualized head model. We have shown with both simulations and real epileptic spike data that standardized and individual BEM models are comparable, that both BEM models delivered improved dipole accuracy over spherical head models, particularly for sources near the base of the brain. We believe that these results support the routine use of standardized head models in clinical source modeling as a substitute for spherical head models. Individualized head models remain the 'gold standard' for source modeling, but when they are not available or practical, a standardized realistic head model is a good compromise.

The sBEM models do not show the systematic localization errors of the spherical approximations, but feature significantly better localization accuracy as compared to individual BEM models. The computational performance of our implementation is about a factor of 60 slower than using analytical spherical models, but is still sufficient for most applications. By linear interpolation of a precomputed leadfield matrix of the standardized BEM model (Yvert et al., 2001) the computational speed could still be increased (work in progress). The sBEM approximation offers an easier and faster access to realistically shaped volume conductor models as compared to individual models and relieves the use of individual 3D MRI data for the BEM model setup. 


\section{Appendix A. Coordinate system matching}

The shift vector $\underline{S}$ and the rotation matrix $\underline{M}$, that are needed to transform points (e.g. the electrode positions) from their original coordinate system to their PAN representation, can easily be achieved from their original landmark coordinates $\underline{L}_{0}, \underline{R}_{\mathrm{o}}$ and $\underline{N}_{\mathrm{o}}$ (left and right preauricular point and nasion). The shift vector $\underline{S}$ to the PAN origin, that has to be applied first, is simply the center between both preauricular points:

$\underline{S}=\left(\underline{R}_{0}+\underline{L}_{0}\right) 0.5$

The $x$-axis direction in the original coordinate system is:

$\underline{X}_{\mathrm{o}}=\left(\underline{R}_{\mathrm{o}}-\underline{L}_{\mathrm{o}}\right) /\left|\left(\underline{R}_{\mathrm{o}}-\underline{L}_{\mathrm{o}}\right)\right|$

with

$\left|\left(\underline{\mathrm{R}}_{\mathrm{o}}-\underline{\mathrm{L}}_{\mathrm{o}}\right)\right|=\left(\left(\underline{R}_{o}-\underline{L}_{\mathrm{o}}\right)\left(\underline{R}_{o}-\underline{L}_{\mathrm{o}}\right)\right)^{1 / 2}$

The $y$-axis is perpendicular to the $x$-axis and points towards the nasion, so it can be calculated from the normal component of the connection from the PAN origin to the nasion:

$\underline{Y}_{\mathrm{o}}=\underline{U}|\underline{U}|$

with

$\underline{U}=\left(\underline{N}_{\mathrm{o}}-\underline{S}\right)-\left(\left(\underline{N}_{\mathrm{o}}-\underline{S}\right) \underline{X}_{\mathrm{o}}\right) \underline{X}_{\mathrm{o}}$

The $z$-axis is finally calculated from the cross-product of the $x$ - and $y$-axes:

$\underline{Z}_{0}=\underline{X}_{o} \times \underline{Y}_{0}$

Combining the 3 basis vectors (as column vectors) to a matrix $A$ results in a rotation matrix that transforms points from the PAN to the shifted original coordinate system. The rotation matrix $M$ that converts points from the shifted original to the PAN system is simply the inverse of this matrix $\underline{\underline{A}}$ :

$\underline{\underline{A}}=\left[\underline{X}_{0} \underline{Y}_{0} \underline{Z}_{0}\right] \quad \underline{\underline{M}}=\underline{\underline{A}}^{-1}=\underline{\underline{A}}^{T}$

So finally the combined transformation to transform a point $P_{\mathrm{o}}$ from the original to the PAN coordinate system reads:

$\underline{P}_{\mathrm{p}}=\underline{\underline{M}}\left(\underline{P}_{\mathrm{o}}-\underline{S}\right)$

The inverse transformation is:

$\underline{P}_{o}=\underline{\underline{M}}^{-1} \underline{P}_{\mathrm{p}}+\underline{S}=\underline{\underline{A}} \underline{P}_{\mathrm{p}}+\underline{S}$

\section{Appendix B. Linear interpolation of the electrode potentials}

Let $R_{i}(i=1 \ldots 3)$ be the node positions of the skin triangle net, then the center position $\underline{R}_{\mathrm{c}}$ of this triangle is given by (Fig. 3):

$\underline{R}_{c}=\left(\underline{R}_{1}+\underline{R}_{2}+\underline{R}_{3}\right) / 3$

For an electrode at position $\underline{R}_{\mathrm{e}}$ the triangle (index $n$ ) with the smallest distance $\left|\underline{R}_{\mathrm{e}}-\underline{R}_{\mathrm{c} n}\right|$ is searched. The edge vectors of the closest triangle are calculated:

$\underline{E}_{12}=\underline{R}_{2}-\underline{R}_{1} \quad \underline{E}_{23}=\underline{R}_{3}-\underline{R}_{2} \quad \underline{E}_{31}=\underline{R}_{1}-\underline{R}_{3}$

Finally the electrode position is projected to the triangles area by using the triangle normal $N$ :

$\underline{N}=\underline{E}_{12} \times \underline{E}_{31}$

The projected position $\underline{R}_{\mathrm{p}}$ is:

$\underline{R}_{\mathrm{p}}=\underline{R}_{\mathrm{e}}+\underline{N}\left(\left(\underline{R}_{\mathrm{e}}-\underline{R}_{c} \underline{N}\right) / \underline{N N}\right.$

The weights $w_{i}(i=1 . .3)$ for the potential values $V_{i}$ at the triangle nodes are computed by linear interpolation from the projected electrode position. The projected electrode position splits the triangle into 3 sub-triangles, their areas $A_{i j}$ are proportional to the cross products of the connecting lines from the projected electrode position to the triangle nodes $\underline{C}_{\mathrm{p} i}$ and the triangle edges $\underline{E}_{i j}$. The total area of the triangle $A$ is proportional to the sum of the areas of the 3 sub-triangles $A_{i j}$ :

$\underline{C}_{\mathrm{p} i}=\underline{R}_{i}-\underline{R}_{\mathrm{p}}$

$A_{i j} \alpha\left|\underline{C}_{\mathrm{p} i} \times \underline{E}_{i j}\right| \quad A \alpha \Sigma A_{i j}$

The weighting factors $w_{i}$ are given by the ratios of the corresponding sub-triangle areas to the total area of the triangle:

$w_{1}=A_{23} / A \quad w_{2}=A_{31} / A \quad w_{3}=A_{12} / A$

So finally the interpolated potential $V_{\mathrm{e}}$ at the (projected) electrode position is given by the weighted sum of the node potentials $V_{i}$ :

$V_{\mathrm{e}}=\Sigma w_{i} V_{i}$

Since the electric potential $V_{i}$ at a node $i$ is computed from a multiplication of the corresponding row $\underline{T}_{i}$ of the transfer matrix $\underline{T}$ (Eq. (3)) with the homogeneous potential column vector $\underline{\bar{V}}_{0}$, a new compressed transfer matrix for the projected electrode positions can be set up:

$\underline{T}_{\mathrm{e}}=\Sigma w_{i} \underline{T}_{i}$

With the much smaller transfer matrix $\underline{\underline{T}}_{\mathrm{e}}$ consisting of rows $\underline{T}_{\mathrm{e} k}$ for all electrodes $k$, the forward calculation of the potentials at all electrode positions (column vector $\underline{V}_{\mathrm{e}}$ ) simplifies to:

$\underline{V}_{e}=\underline{T}_{\mathrm{e}} \underline{V}_{0}$ with $\underline{V}_{0}$ from Eq. (4).

\section{References}

van Burik MJ, Peters MJ. Estimation of the electric conductivity from scalp measurements: feasibility and application to source localization. Clin Neurophysiol 2000;111:1514-1521.

Cuffin BN. Effects of head shape on EEGs and MEGs. IEEE Trans Biomed Eng 1990;37:44-52.

Ferguson AS, Zhang X, Stroink G. A complete linear discretization for 
calculating the magnetic field using the boundary element method. IEEE Trans Biomed Eng 1994;41:455-460.

Ferguson AS, Stroink G. Factors affecting the accuracy of the boundary element method in the forward problem I: calculating surface potentials. IEEE Trans Biomed Eng 1997;44:1139-1155.

Fletcher DJ, Amir A, Jewett DL, Fein G. Improved method for computation of potentials in a realistic head shape model. IEEE Trans Biomed Eng 1995;42:1094-1104.

Frijns JH, de Snoo SL, Schoonhoven R. Improving the accuracy of the boundary element method by the use of second-order interpolation functions. IEEE Trans Biomed Eng 2000;47:1336-1346.

Fuchs M, Drenckhahn R, Wischmann HA, Wagner M. An improved boundary element method for realistic volume-conductor modeling. IEEE Trans Biomed Eng 1998;45:980-997.

Fuchs M, Wagner M, Kastner J. Boundary element method volume conductor models for EEG source reconstruction. Clin. Neurophysiology 2001;112:1400-1407.

Geddes LA, Baker LE. The specific resistance of biological material, a compendium of data for the biomedical engineer and physiologist. Med Biol Eng 1963;5:271-293.

Geselowitz DB. On bioelectric potentials in an inhomogeneous volume conductor. Biophys J 1967;7:1-17.

Hämäläinen MS, Sarvas J. Realistic conductivity geometry model of the human head for interpretation of neuromagnetic data. IEEE Trans Biomed Eng 1989;36:165-171.

Law SK. Thickness and resistivity variations over the upper surface of the human skull. Brain Topogr 1993;6:99-109.

Meijs JWH, Weier OW, Peters MJ, van Oosterom A. On the numerical accuracy of the boundary element method. IEEE Trans Biomed Eng 1989;36:1038-1049.

de Munck JC. A linear discretization of the volume conductor boundary integral equation using analytically integrated elements. IEEE Trans Biomed Eng 1992;39:986-990.

Musha T, Okamoto Y. Forward and inverse problems of EEG dipole localization. Crit Rev Biomed Eng 1999;27:189-239.

Nelder JA, Mead R. A simplex method for function minimization. Comput J 1965;7:308-313.

Oostendorp TF, van Oosterom A. Source parameter estimation in inhomogeneous volume conductors of arbitrary shape. IEEE Trans Biomed Eng 1989;36:382-391.

van Oosterom A, Strackee J. The solid angle of a plane triangle. IEEE Trans Biomed Eng 1983;30:125-126.

Sarvas J. Basic mathematical and electromagnetic concepts of the biomagnetic inverse problem. Phys Med Biol 1987;32:11-22.

Schlitt HA, Heller L, Aaron R, Best E, Ranken DM. Evaluation of boundary element methods for the EEG forward problem: effect of linear interpolation. IEEE Trans Biomed Eng 1995;42:52-58.

Wagner M, Fuchs M, Wischmann HA, Ottenberg K, Dössel O. Cortex segmentation from 3D MR images for MEG reconstructions. In: Baumgartner C, Deecke L, Stroink G, Williamson SJ, editors. Biomagnetism: fundamental research and clinical applications, Amsterdam: Elsevier Science/IOS Press, 1995. pp. 352-356.

Wagner M, Fuchs M, Drenckhahn R, Wischmann HA, Köhler T, Theissen A. Automatic generation of BEM and FEM meshes. Neuroimage 1997;5:389.

Yvert B, Bertrand O, Echallier JF, Pernier J. Improved dipole localization using local mesh refinement of realistic head geometries. Electroenceph clin Neurophysiol 1995;95:381-392.

Yvert B, Crouzeix-Cheylus A, Jacques Pernier J. Fast realistic modeling in bioelectro-magnetism using lead-field interpolation. Hum Brain Mapp 2001;14:48-63. 